\title{
El comportamiento del precio del petróleo Brent y los ingresos tributarios: un desafío fiscal para el Gobierno colombiano, 2007-2020
}

\section{The behavior of the price of Brent oil and tax revenues: A fiscal challenge for the Colombia` Government 2007-2020}

\section{O comportamento do preço do petróleo Brent e as receitas fiscais: um desafio fiscal para o Governo de Colombia 2007-2020}

Luisa María Hoyos Franco*

Actualmente cursa la especialización en Econometría, Universidad Externado de Colombia; economista de la Universidad de La Salle. Coordinadora asistente en el Departamento de Derecho Fiscal, Universidad Externado de Colombia. luisamhf@gmail.com / https://orcid.org/0000-0002-7593-6597. Dor: https://doi.org/10.18601/16926722.n19.05 


\title{
Resumen
}

Este artículo tiene por objetivo analizar el comportamiento del precio promedio trimestral del petróleo de referencia Brent y su relación con los ingresos tributarios entre los años 2007-I y 2020-I. La metodología implementada consistió en realizar modelos econométricos para validar el test de Chow con el fin de determinar una desviación en la tendencia del precio del petróleo. Como resultado se evidenció la existencia de tres cambios estructurales en el primer trimestre de los años 2012, 2014 y 2017. Adicionalmente, los resultados de los tres modelos reflejaron que, a mayores ingresos recibidos por parte de las empresas petroleras, el gobierno no tiene incentivos para gravarlas ya que tienen una variedad de alivios tributarios en el momento de liquidar sus impuestos.

Palabras clave: precio del petróleo Brent, cambio estructural, ingresos tributarios.

\begin{abstract}
This article aims to analyze the behavior quarterly of the average price of oil of reference Brent and your relation with the tax revenues between the years 2007-I and 2020-I. The methodology implemented consists in realize econometric models for validating the test of Chow to determinate a deviation of the trend in the price of oil. As a result, was evidence the existence of three structural changes in the first quarter of the years 2012,2014 and 2017, additionally, the results of the three models reflected that as of a major tax collection received on the part of oil companies, the government has no incentive for the tax it because has a variety of tax breaks in the moment of settling their taxes.
\end{abstract}

Key words: Price of oil Brent, estructural change, tax revenues.

\section{Resumo}

Este artigo tem como objetivo analisar o comportamento do preço médio trimestral do petróleo de referência Brent e sua relação com as receitas fiscais entre os anos de 2007-I e 2020-I. A metodologia implementada consistiu na realização de modelos econométricos para validação do teste de Chow com o objetivo de determinar um desvio na tendência do preço do petróleo. Como resultado, foi evidenciada a existência de três mudanças estruturais no primeiro trimestre dos anos de 2012, 2014 e 2017. Adicionalmente, os resultados dos três modelos refletiram que os maiores rendimentos auferidos das petroleiras, o governo não tem incentivos para tributá-las. pois eles têm uma variedade de incentivos fiscais para se beneficiar ao pagar seus impostos.

Palavras chave: Preço do petróleo Brent, mudança estrutural, receita tributária. 


\section{Introducción}

Desde mediados de los años cincuenta, el petróleo se ha convertido en la fuente de energía más importante del mundo. Debido a la dinámica que se ha construido alrededor de la industria petrolera, de acuerdo con Gómez (2015), la tensión en el mercado genera susceptibilidad en las economías de todo el mundo ante choques de oferta o de demanda; por lo anterior, cuando se presenta algún cambio o ajuste, por mínimo que este sea, los choques en el precio del crudo impactan de diferentes maneras a las economías, por ejemplo, en economías importadoras netas de petróleo, los choques pueden desencadenar en una recesión, y, por el contrario, en los países productores, los choques tienen el potencial de generar bonanzas económicas que, bien administradas, incitan crecimiento y desarrollo.

En consecuencia, el análisis económico fundamental muestra que la complejidad de la evolución de los precios del petróleo en el mundo puede explicarse a través de las relaciones dinámicas y cambiantes entre los países productores, las compañías petroleras, las refinerías individuales, los países importadores y los especuladores (Yu et al., 2008); al igual que la presencia de eventos económicos, sociales y políticos (Álarez-Ramírez et al., 2008) y naturales, como los desastres causados por los huracanes Dennis, Katrina y Rita. Así mismo, los precios del petróleo dependen de los niveles de inventarios y cambios en las reservas, la capacidad de producción y refinación y los avances tecnológicos en todos los pasos de la cadena de producción. (Velásquez et al., 2009, p. 249)

En Colombia, luego de varios años en los que el sector sufrió descrédito por los constantes cambios en las condiciones de los contratos de exploración y explotación que, finalmente, terminaron ahuyentando la inversión extranjera, en el año 2003 el Gobierno de turno reformó la Empresa Colombiana de Petróleos (Ecopetrol), con el objetivo de internacionalizarla y, por ende, volverla más competitiva a nivel mundial en la industria de los hidrocarburos.

Con la expedición del Decreto 1760 del 26 de junio de 2003, se modificó la estructura orgánica de la compañía, transformación que permitió que se incrementaran las actividades exploratorias y se adquiriera una nueva dinámica en el país para las actividades petroleras. Este decreto también creó la Agencia Nacional de Hidrocarburos (ANH), cuya función principal es la de administrar todos los recursos hidrocarburíferos del país; a partir de ese momento, la exploración creció en más del triple, pasando de 28 pozos exploratorios en 2003 a 606 en 2019.

Por lo anterior, según la Contraloría General de la República, "los efectos de los bajos precios se perciben en una reducción de los impuestos recibidos del sector petrolero, especialmente de Ecopetrol que es la petrolera más grande en Colombia, y de los dividendos que dejará de recibir de esta por ser el Estado su accionista mayoritario" (2016,p. 1). De ahí que, surge la siguiente pregunta: ¿cómo ha sido el comportamiento del precio 
promedio trimestral del petróleo de referencia Brent y cuál es su relación con los ingresos tributarios entre los años 2007 y 2020 ?

Ahora bien, con el fin de hacer un diagnóstico que permita identificar el comportamiento del precio promedio trimestral del petróleo de referencia Brent entre los años 2007-I y 2020-I y su relación con los ingresos tributarios, se utiliza el test de Chow a fin de determinar una desviación de la tendencia estructural en el precio del petróleo de referencia Brent.

Cabe resaltar que, a raíz de los modelos de regresión lineal implementados para llevar a cabo el test, se pudo conocer la relación existente entre el precio del crudo y los ingresos tributarios. De esta manera, el presente artículo se divide en cinco secciones: la primera pretende realizar una revisión histórica de los antecedentes que permitan acercarse al debate sobre el comportamiento del precio del crudo Brent y su relación con los ingresos tributarios; la segunda desarrolla la metodología; la tercera presenta los hechos estilizados, teniendo en cuenta que las cifras del recaudo y de los beneficios tributarios del sector de hidrocarburos estaban desactualizadas, el presente trabajo pretende actualizarlas; la cuarta muestra los resultados obtenidos, donde se calcula el test de Chow con el fin de determinar el cambio estructural en el precio y, de tal manera, conocer la relación entre este commoditie y los ingresos tributarios; finalmente, en la quinta, se presentan las conclusiones.

\section{Estado del arte}

Con ocasión de exponer la literatura académica más relevante acerca del comportamiento del precio del petróleo Brent y la relación que este commoditie tiene con los ingresos tributarios en Colombia, este apartado tiene como fin resumir las conclusiones de los estudios empíricos más relevantes, que exploran los temas antes mencionados. Por lo anterior, es imprescindible mencionar los autores más destacados.

El estudio del análisis económico fundamental pone en evidencia que el comportamiento de los precios del crudo en el mundo se puede explicar por acontecimientos económicos, sociales y políticos; dicha premisa fue demostrada mediante la investigación realizada por Álvarez-Ramírez et al. (2008), donde a través de pruebas de variaciones temporales del exponente de Hurst indican que, en horizontes largos, el mercado del petróleo crudo es consistente con la hipótesis del mercado eficiente.

Dada la importancia del petróleo como fuente primaria de energía de la cual depende la sociedad, el estudio de la dinámica de los precios del crudo fue objeto de estudio por Velásquez-Olaya et al. (2009), donde a partir de algoritmos de búsqueda basados en un particionamiento recursivo para detectar los puntos de ocurrencia de cambios estructurales en la tendencia, lograron identificar tres cambios estructurales estadísticamente significativos del precio promedio mensual del petróleo de referencia WTI entre 1986:1 y 2008:8, los cuales son explicados por eventos históricos específicos; el primer cambio en la tendencia según los autores, se explica en parte por varios sucesos: 
el crecimiento de la producción de la Organization of the Petroleum Exporting Countries (OPEC) y el uso de distintas fórmulas para la valoración del petróleo; y la segunda corresponde al segundo semestre de 1990 y primer semestre de 1991, período durante el cual se dio la invasión de Kuwait por parte de Iraq, y la guerra del Golfo Pérsico. (Velásquez et al., 2009, p. 256)

A partir de los planteamientos mencionados, se hará énfasis especialmente en los estudios enfocados en la incidencia que el precio del petróleo tiene en los ingresos tributarios, por ende, se destaca un estudio realizado por el Banco Interamericano de Desarrollo (BID) (2013), donde se determina la vulnerabilidad de los ingresos públicos provenientes de hidrocarburos ante cambios en el precio internacional del petróleo a través del cálculo de elasticidades. Por lo anterior, la institución argumenta que:

Los dividendos e impuestos pagados por Ecopetrol representan ingresos importantes para la nación, y aunque mostraron una tendencia creciente hasta 2008, disminuyeron en 2010. Esto se debe a que en el año 2009 se pagaron los dividendos y el impuesto de renta causados en 2008, año en el que los resultados financieros de Ecopetrol fueron particularmente buenos, mientras que en 2010 se pagaron los dividendos y el impuesto de renta causados en 2009, cuando dichos resultados no fueron tan positivos. (p. 17)

Para el caso de los países productores de petróleo, como Colombia, una época de bonanza refleja mayores ingresos tributarios recaudados por parte del sector de hidrocarburos, de ahí que, determinados impuestos tienen mayor participación que otros; en razón de lo antes expuesto, se trae a colación el estudio realizado por la Unidad de la Planeación Minero Energética (UPME) (2015), por su evaluación sobre la contribución económica del sector de hidrocarburos al Estado, donde resalta la importancia del sector petrolero en Colombia; la UPME hace énfasis en los ingresos tributarios recaudados por los hidrocarburos y argumenta que, si se desagrega la contribución fiscal del sector petrolero, se observa que los aportes más importantes provienen del impuesto de renta y de los dividendos de Ecopetrol. Los demás impuestos, incluyendo el IVA, el IVA externo y los aranceles representan montos relativamente pequeños que, en general, se mantienen por debajo del $1 \%$ de los ingresos corrientes del gobierno (UPME, 2015, p. 12).

En la misma línea de pensamiento, se destaca el trabajo realizado por la Contraloría General de la República (2016), que investigó el impacto de la crisis petrolera en los ingresos del Gobierno nacional central (GNC). En dicho trabajo se señaló que la coyuntura presentada por el descenso en las cotizaciones del petróleo desde mediados de 2014 ha impactado las finanzas públicas en los órdenes nacional y territorial, vía menores ingresos.

En términos de ingresos para el Gobierno Nacional, las rentas asociadas con los hidrocarburos derivadas de los pagos de impuesto de renta y CREE que hacen las empresas del sector, y los dividendos que paga Ecopetrol a la Nación, han representado en 
promedio el $17 \%$ del total de sus ingresos durante los últimos tres años, p. 13 Marco

Fiscal de Mediano Plazo 2015. (Contraloría General de la República, 2016, p. 2)

La magnitud de los ingresos marginales del petróleo en Colombia también ha sido objeto de estudio usando diversas metodologías como la implementada por el Ministerio de Hacienda y Crédito Público (Valencia et al., 2018) en su análisis basado en un enfoque de hoja de balance y un modelo vAR con parámetros cambiantes en el tiempo y volatilidad estocástica (TVP- VAR-SV), donde concluyen que:

Los efectos de los cambios en el precio del petróleo en los ingresos del Gobierno Nacional Central fueron asimétricos, no lineales y no constantes a lo largo del tiempo, siendo mayores cuando los precios caen que cuando suben, y en los bajos niveles de precios del petróleo, adicionalmente, encontraron que ante un aumento/caída de 1 USD en el precio del petróleo se incrementan/reducen los ingresos tributarios en una magnitud de 175 miles de millones de pesos ( $\mathrm{mm}$ ), en promedio, con una mayor magnitud en el efecto cuando los precios caen. (p. 21)

Partiendo de una visión global de los análisis descritos, se pone en evidencia que el comportamiento del precio del petróleo en el mundo se ve reflejado en acontecimientos económicos, políticos y sociales que pueden ocasionar una baja o subida inesperada de los precios dado un choque, bien sea de oferta o de demanda; por esto es que al precio del crudo se le atribuye una gran volatilidad, razón por la cual expertos en la materia han modelado y pronosticado la tendencia y la volatilidad de dicho commoditie, lo que ha llevado a explicar las causas de la variación en el precio del petróleo; no obstante, aunque estos análisis son indispensables, no explican en su totalidad las causas de las variaciones del precio ya que excluyen variables que no se observan tan fácil, por ejemplo, factores de oferta, como la tecnología o las políticas de regulación o de demanda como las expectativas del precio; por tanto, frente al escenario descrito anteriormente el presente documento busca determinar con mayor detalle el comportamiento de los precios del petróleo Brent, a fin de captar la influencia de los hechos que indujeron a un cambio en la tendencia de los precios del crudo y su relación en los ingresos tributarios.

A causa de lo anterior, la dinámica que ha venido viviendo el país muestra que existe una relación directa entre el precio del petróleo y los ingresos tributarios, ya que se evidencia un mayor recaudo cuando el precio presenta un crecimiento sostenido, contrario a lo que sucede si el precio del crudo manifiesta una tendencia a la baja dado que se ve reflejado en una disminución de los ingresos para el Gobierno, por consiguiente, según la Contraloría General de la República (2016), en promedio, entre 2013 y 2014, el 86\% de los ingresos del GNC se derivaron de los tributos, razón por la cual el impuesto sobre la renta, el IVA y otros tributos perciben mayor recaudo por parte de las empresas petroleras. 
Por lo anterior, la comunidad académica y otros sectores discuten si las compañías vinculadas al sector de hidrocarburos contribuyen al Estado con un alto pago de impuestos; al respecto sobresalen investigaciones como la llevada a cabo por el economista Luis Álvaro Pardo, quien en entrevista con la revista Semana afirma que las empresas no pagan tantos impuestos como deberían, especialmente en los sectores bancario y de minas y petróleos, y que, con cada reforma, lo que se ahorran sube como espuma. Por esta razón, se busca determinar la relación que surge entre en el precio del crudo y los ingresos tributarios, por lo que el presente trabajo actualiza las estadísticas del impuesto de renta pagado por Ecopetrol a la nación y contextualiza los beneficios tributarios otorgados al sector de hidrocarburos.

\section{Metodología}

\section{A. Datos utilizados}

Las variables utilizadas en la presente investigación para abordar el objetivo propuesto corresponden al precio promedio trimestral del petróleo crudo de referencia Brent dólares por barril, en adelante (bl), que se transa en el electrónico Intercontinental Exchange (ICE) y en el New York Mercantile Exchange (NYMEX), que es un referente para valorar contratos de futuros y opciones financieras; adicional al precio del petróleo se usaron estadísticas de recaudo trimestrales. Es preciso señalar que los datos antes mencionados fueron obtenidos de la página web de Investing y de la Dirección de Impuestos y Aduanas Nacionales (DIAN), respectivamente, las series analizadas corresponden al periodo comprendido entre los años 2007-I y 2020-I.

Con el fin de analizar el comportamiento del precio promedio trimestral del petróleo de referencia Brent entre los años 2007-I y 2020-I y su relación con los ingresos tributarios, se utiliza el test de Chow para determinar una desviación de la tendencia estructural en el precio del petróleo; cabe resaltar que, a raíz de los modelos de regresión lineal implementados para llevar a cabo el test, se conoció la relación existente entre el precio del crudo y los ingresos tributarios, donde la producción de petróleo es una fuente importante de generación de ingresos del Estado; además, se concede un papel protagónico a la política fiscal en el sentido de que tiene la potestad de controlar el recaudo tributario de dichos recursos. Esta metodología permite determinar con mayor detalle el comportamiento de los precios del petróleo Brent, a fin de captar la influencia de los hechos que indujeron a un cambio en la tendencia de los precios del crudo y su relación con los ingresos tributarios.

El desarrollo metodológico consta de tres apartados: primero, se explica el test de Dickey Fuller para determinar la estacionariedad de las series; después, siguiendo a Quintana y Mendoza (2010) se realiza la prueba de Johansen, con el fin de determinar la cointegración de las variables y, finalmente, el test de Chow en su estructura general sigue a Wooldridge (2015) para determinar si existe un cambio estructural en el precio del crudo. 


\section{Test de Dickey-Fuller}

En primera instancia, se realizó el test de Dickey-Fuller que, según Wooldridge (2010), es una forma simple de probar la hipótesis nula de no cointegración frente a la alternativa de cointegración, una de las cuales está basada en la prueba de raíz unitaria de Dickey-Fuller a los residuales de una regresión estática.

Para aplicar dichas pruebas se parte de un modelo de camino aleatorio puro, pero se resta el término autorregresivo de los dos lados:

$$
\begin{gathered}
y_{t}-y_{t-1}=\phi y_{t-1}-y_{t-1}+u_{t} \\
\Delta y_{t}=\gamma y_{t-1}+u_{t}
\end{gathered}
$$

Donde:

$$
\begin{gathered}
\Delta y_{t}=y_{t}-y_{t-1} \\
\gamma(\phi-1)
\end{gathered}
$$

De esta manera, con una raíz unitaria $\phi=1$, el parámetro $y=0$. Del modelo propuesto resulta entonces tentador efectuar la prueba de raíz unitaria aplicándole mínimos cuadrados ordinarios al modelo en primeras diferencias y sin constante. El coeficiente estimado del término autorregresivo se podría someter a la prueba usual de significancia estadística $t$ para contrastar la hipótesis nula de raíz unitaria contra la alternativa de estacionariedad:

$$
\begin{aligned}
& H_{0}: y=0 \text { por consiguiente } \phi=1 \\
& H_{A}: y<0 \text { por consiguiente } \phi<1
\end{aligned}
$$

La prueba esbozada aquí permite afirmar que, de no existir evidencia en contra de la hipótesis nula, la serie no será estacionaria y seguirá una caminata aleatoria pura (Quintana y Mendoza, 2016, pp. 179-180).

\subsection{Test de Johansen}

No obstante, se procedió cointegrando las series mediante el test de Johansen, como lo mencionan Quintana y Mendoza (2010). Con base en dicho método es posible probar tanto el orden de integración de un conjunto de variables, como la existencia de cointegración entre las mismas; una vez las series se encuentran cointegradas se procede a realizar el test de Chow. 


\subsection{Test de Chow}

En cuanto a la metodología para determinar el cambio estructural, como argumenta el autor, en el modelo general con $\kappa$ variables explicativas y un intercepto, se tienen dos grupos: $g=1$ y $: g=2$. Se desearía probar si los interceptos y las pendientes son iguales en los dos grupos. Este modelo se expresa como

$$
\gamma=\beta_{g, 0}+\beta_{g, 1} \chi_{1}+\beta_{g, 2} \chi_{2}+\ldots+\beta_{g, k} \chi_{k}+u
$$

para $g=1$ y $g=2$.

La hipótesis de que cada beta en [5] es igual en los dos grupos incluye $\kappa+1$ restricciones. La idea clave es que la suma de los residuales cuadrados del modelo no restringido puede obtenerse de dos regresiones separadas, una para cada grupo: sea $S R C_{1}$ la suma de residuales cuadrados obtenida estimando [5] para el primer grupo (aquí intervienen $n_{1}$ observaciones); sea $S R C_{2}$ la suma de residuales cuadrados obtenida de la estimación del modelo empleando el segundo grupo (aquí intervienen $n_{2}$ observaciones). Ahora, la suma de los residuales cuadrados para el modelo no restringido es simplemente $S R C_{n r}=S R C_{1}+S R C_{2}$ . La suma restringida de residuales cuadrados es sencillamente la SRC de juntar los grupos y estimar una sola ecuación, $S R C_{p}$. Una vez que se tienen estas, se calcula el estadístico F como de costumbre:

$$
F=\frac{\left[S R C_{p}-\left(S R C_{1}+S R C_{2}\right)\right]}{S R C_{1}+S R C_{2}} \cdot \frac{n-2(k+1)}{k+1},
$$

Donde $n$ es la cantidad total de observaciones. A este estadístico F particular se le conoce en econometría como estadístico de Chow, la prueba de Chow es simplemente una prueba F. (Wooldridge, 2015, p. 245).

\section{Hechos estilizados}

El presente apartado se compone de cuatro secciones. En la primera se exponen los principales hitos históricos que han influido en un cambio de la tendencia del precio del petróleo Brent; en la segunda se presentan los hallazgos más relevantes en la medida en que un cambio en el precio promedio del petróleo impacta en los ingresos tributarios; en la tercera, se relacionan los impuestos pagados por Ecopetrol a la nación y, en la cuarta, se contextualiza el monto y el costo fiscal de los beneficios tributarios y otras deducciones del sector de hidrocarburos. 
Para comenzar, en primera instancia se hace referencia al comportamiento del precio del crudo en los últimos 13 años y los principales hechos históricos que marcaron algunos de sus crecientes y decrecientes.

En la figura 1 se observa la evolución en el tiempo del precio del petróleo promedio de referencia Brent para el periodo 2007-I y 2020-I. La inspección visual demuestra la presencia de varias tendencias lineales mayores, las crecientes corresponden a: segundo y tercer trimestre de 2008, 2011, 2012, 2013 y 2014 hasta el tercer trimestre; mientras que las decrecientes se presentaron en: cuarto trimestre del 2008, 2015, 2016, 2017 y primer trimestre del 2020; principalmente, dichas fluctuaciones rápidas en los precios pueden asociarse a diferentes sucesos históricos que se mencionarán a continuación.

Para el año 2007, el precio promedio del crudo era de US\$74,68 (bl), su comportamiento tendía al alza ya que según Gómez (2015), en su momento el gobierno había conseguido consolidar, organizar y hacer más competitivo y atractivo el sector petrolero; sin embargo, para el año 2008, a raíz de la crisis financiera, los precios empezaron a disminuir a partir del cuarto trimestre, con lo cual se vieron reflejados los efectos adversos de la crisis. Expertos en la materia señalan: "La disminución acelerada de la cotización del crudo ya se había observado: durante la crisis financiera de 2008, su precio se redujo $70 \%$ en seis meses. Algo similar se observó en 1985 y 1997, cuando en un semestre disminuyó $60 \%$ y $35 \%$, respectivamente" (Banco de la República, 2016, p. 2).

Así las cosas, para el año 2009 el precio promedio del crudo anual fue de US\$64,09 bl, después de la contracción de la demanda mundial de 2008, para el año 2010 los precios empiezan a recuperarse y llegan a los U\$ 80,65 bl, superando el promedio anual respecto del año anterior; como se puede observar en la figura 1 para el año 2011 se observa una recuperación significativa donde el precio promedio alcanzó los US \$118,04 bl aumento que no se observaba desde el año 1864, dicho acontecimiento se atribuye al incremento del 2,5\% en el consumo global de energía, donde China representó el $71 \%$ del crecimiento en el consumo de energía, sumado a esto, según Carcar (2012) se produjo el mayor aumento en producción de la OPEP desde el año 2008, por ende, dicho comportamiento positivo de los precios se vio reflejado durante los años 2012, 2013 y hasta el tercer trimestre del año 2014.

Inesperadamente, a partir del cuarto trimestre del año 2014, el precio disminuyó aproximadamente un poco más de US\$30 bl respecto del trimestre anterior, dado que, según la Agencia Internacional de la Energía (International Energy Agency - IEA), en 2014 la oferta mundial creció por encima de la demanda, lo que permitió una acumulación de inventarios cercana al millón de barriles diarios. Este exceso se originó principalmente por un incremento, más allá de lo esperado, de la extracción de crudo en Estados Unidos, explicado por la mayor producción de crudos no convencionales, lo cual se hizo viable comercialmente gracias al incremento de los precios y a las favorables condiciones de financiamiento.

Adicionalmente, el Banco de la República señala que este acontecimiento también se originó por una mayor oferta en algunos países del Oriente: "Durante 2014 la oferta de 


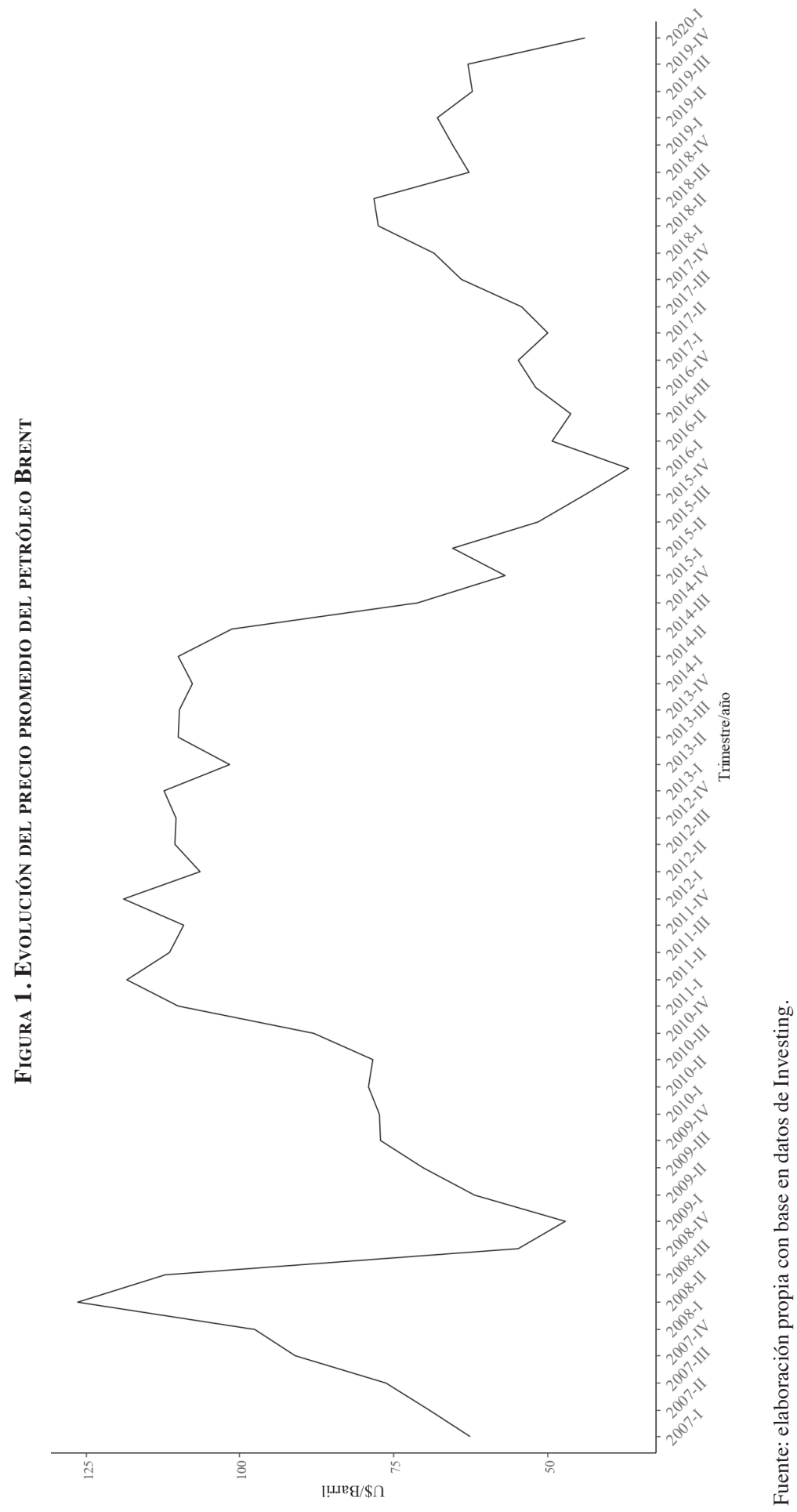

Revista de Derecho Fiscal n. ${ }^{\circ} 19$ • julio-diciembre de 2021 • pp. 109-131 
crudo también se originó gracias al incremento inesperado de la extracción de hidrocarburos en países con altos riesgos geopolíticos, tales como Irak, Libia y Rusia, los cuales, al no materializarse, dejaron al mercado con una oferta importante de crudo" (Banco de la República, 2016,p. 3).

Para el año 2015, el precio promedio anual continuó descendiendo hasta alcanzar los US\$43,82 bl, lo anterior se explica, según el Banco de la República, por:

La persistente disminución de la demanda mundial, a esto se suma la decisión de la OPEP de no recortar su producción, pese a la caída de la cotización internacional del crudo. Dicha decisión, junto con la disminución unilateral de los precios por parte de algunos de los principales productores de esta organización pretendería mantener la participación de la OPEP en el mercado mundial de hidrocarburos. (2016, p. 3)

Para terminar, la caída de los precios del crudo del primer trimestre del año 2020 obedece a la contracción de la demanda mundial de petróleo debido a la pandemia decretada por la Organización Mundial de la Salud (OMs) a causa de la pandemia del covid-19.

En la figura 2, a la izquierda se puede observar la evolución en el tiempo del precio promedio del petróleo de referencia Brent, y, a la derecha, los ingresos tributarios entre los años 2007-I y 2020-I. Como se mencionó, en Colombia, al ser un país exportador de petróleo, un descenso en las cotizaciones impacta a las finanzas nacionales y territoriales a través de menores ingresos; a causa de lo anterior, según la Contraloría General de la República, "los efectos de los bajos precios se perciben en una reducción de los impuestos recibidos del sector petrolero, especialmente de Ecopetrol que es la petrolera más grande, y de los dividendos que dejará de recibir de esta por ser el Estado su accionista mayoritario" (2016, p. 1).

Sin embargo, pese a que el precio del petróleo es un factor determinante para la generación de ingresos para la nación, como se puede observar en la figura 2, el recaudo ha venido aumentado continuamente, en gran medida como resultado de las reformas tributarias, como lo hace notar un estudio de la Comisión Económica para América Latina y el Caribe (Cepal):

En las últimas tres décadas el recaudo administrado por la Dirección de Impuestos y Aduanas Nacionales (DIAN) ha crecido en términos reales, donde el recaudo total creció $81 \%$ entre el periodo de reforma del estado y apertura (1985-94) y el de ajuste y crisis (95-2004); y entre este y el de auge primario (2005-2014), el recaudo promedio se expandió $119 \%$. Se puede decir que el recaudo se ha duplicado cada década, y cuadriplicado en 20 años. (2017,p. 9)

A continuación, se detallarán algunos impuestos pagados por Ecopetrol a la nación entre los años 2013 y 2019. 


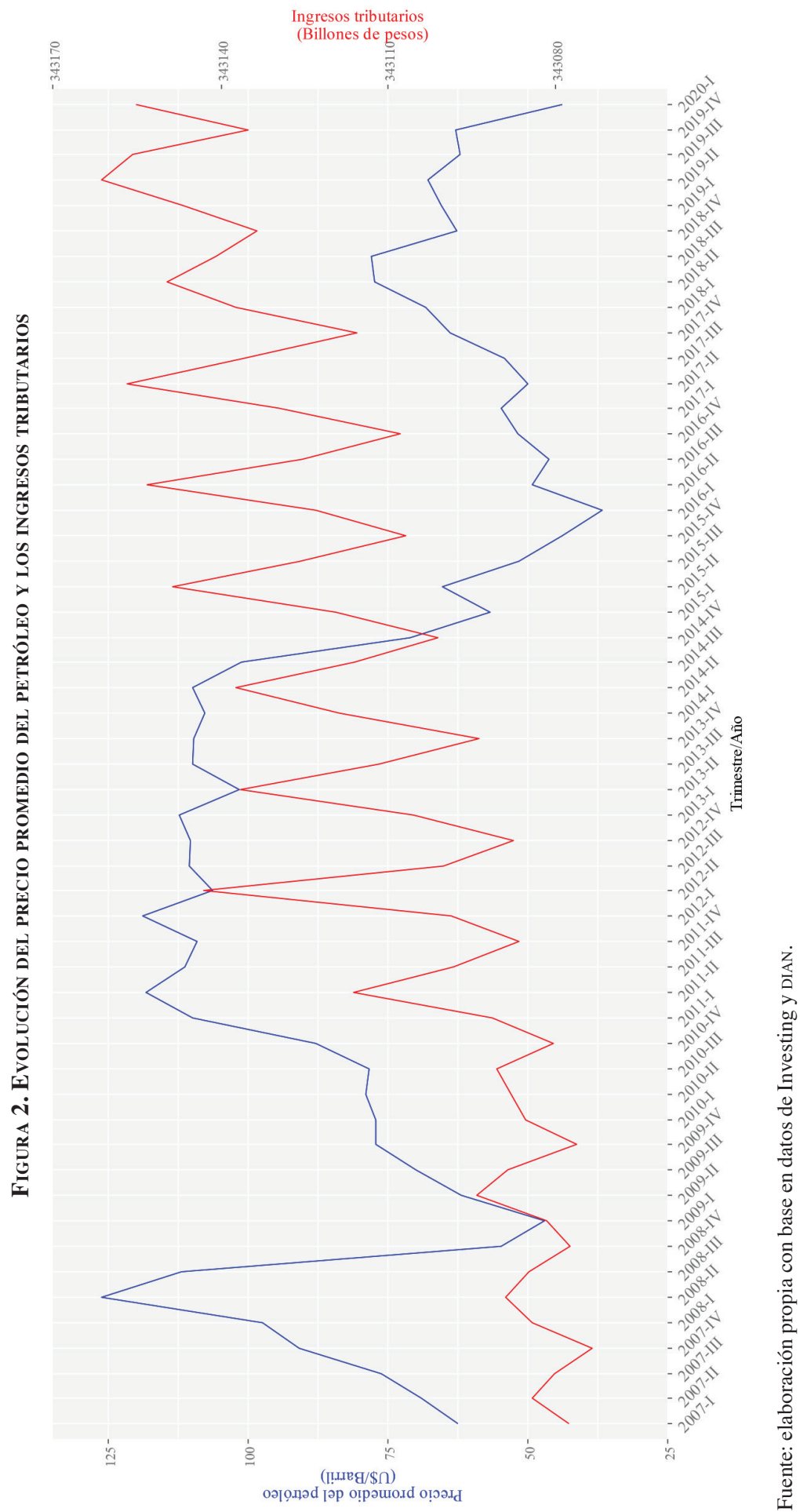


Como se puede observar en la tabla 1, Ecopetrol contribuye a la nación principalmente vía impuesto de renta, otorgándole una gran importancia a este tributo en el régimen tributario colombiano. Es de destacar que para el año 2013 la contribución del impuesto de renta por cuenta de Ecopetrol fue de $\$ 6,8$ billones, el más alto en los últimos 7 años, toda vez que en 2013 se pagaron los tributos causados en 2012, año que fue particularmente bueno para el petróleo, superando un precio promedio anual de US\$111 (bl). Según la Contraloría General de la República (2016), las empresas del sector pagaron en el impuesto de renta $\$ 9,1$ billones, es decir que del total del sector, Ecopetrol pagó $75 \%$. Contrario a la alta contribución del impuesto de renta evidenciado en el año 2013, se observa que en 2018 se dio el aporte más bajo, con \$272 millones donde el precio promedio anual del crudo para el año 2017 fue de US\$55,7 (bl), precio que ciertamente no es favorable para los ingresos de la economía colombiana.

Por otra parte, debido a la entrada en vigencia de la reforma tributaria (Ley 1819 de 2016), la autorretención por renta, figura que reemplazó al impuesto para la equidad CREE, equiparó la afectación del recaudo. No obstante, se observa que los ingresos que Ecopetrol aporta a la nación vía tributos no tienen un crecimiento sostenido y, por el contrario, tienden a disminuir, toda vez que la suma de los impuestos pagados en 2013 fue de $\$ 9,1$ billones y en 2019 de \$6,08 billones, presentando un descenso del $33 \%$.

Tabla 1. Impuestos Pagados por Ecopetrol a la NaCión (MILlones de PeSos CORRIENTES)

\begin{tabular}{|l|c|c|c|c|c|c|c|}
\hline \multicolumn{1}{|c|}{ Concepto } & $\mathbf{2 0 1 3}$ & $\mathbf{2 0 1 4}$ & $\mathbf{2 0 1 5}$ & $\mathbf{2 0 1 6}$ & $\mathbf{2 0 1 7}$ & $\mathbf{2 0 1 8}$ & $\mathbf{2 0 1 9}$ \\
\hline Impuesto de renta & 6.835 .239 & 2.972 .253 & 1.493 .191 & - & 372.438 & 272.763 & - \\
\hline $\begin{array}{l}\text { Impuesto de renta pa- } \\
\text { ra la equidad cREE }\end{array}$ & - & 1.219 .524 & 1.145 .108 & - & - & - & - \\
\hline $\begin{array}{l}\text { Autorretención de Re- } \\
\text { tención para la equi- } \\
\text { dad - CREE }\end{array}$ & 563.320 & 963.120 & 719.575 & 634.400 & 75.130 & - & - \\
\hline $\begin{array}{l}\text { Autorretenciones por } \\
\text { renta }\end{array}$ & 837.628 & 902.634 & 651.671 & 548.792 & 1.407 .033 & 5.094 .734 & 2.172 .632 \\
\hline Subtotal & 8.236 .187 & 6.057 .531 & 4.009 .545 & 1.183 .192 & 1.854 .601 & 5.367 .497 & 2.172 .632 \\
\hline Otros & 935.734 & 938.395 & 841.569 & 4.433 .137 & 3.644 .902 & 3.437 .668 & 3.913 .776 \\
\hline Total nación & 9.171 .921 & 6.995 .926 & 4.851 .114 & 5.616 .329 & 5.499 .503 & 8.805 .165 & 6.086 .408 \\
\hline $\begin{array}{l}\text { Precio petróleo Brent } \\
\text { (promedio anual) }\end{array}$ & 108,4 & 97,5 & 54,4 & 46,0 & 55,7 & 71,6 & 64,6 \\
\hline
\end{tabular}

Fuente: Contraloría General de la República (2016) y Reporte Integrado de Gestión Sostenible 2015 y 2019 Ecopetrol e Investing.

En la tabla 1 se observa que la contribución de Ecopetrol vía impuestos pagados a la nación no es estable debido a la volatilidad en los precios, sin embargo, el papel que los beneficios tributarios juegan en la base gravable en el impuesto de renta de las compañías petroleras representa una importante suma que, al final, se ve reflejada en un aumento del 
costo fiscal para la nación. Como plantea Pardo en su investigación a partir de un análisis de las estadísticas oficiales de las declaraciones de renta de las personas jurídicas: "Aunque la crisis de las finanzas públicas suele atribuirse a la caída de los precios del petróleo desde el 2014, también contribuyeron las billonarias deducciones realizadas por las compañías de minas e hidrocarburos, en un monto promedio de 3.300 millones de dólares anuales en el periodo 2010-2016" (2018, párr. 1).

Para profundizar en la anterior afirmación, a continuación se presentan dos tablas, en la tabla 2 se expone las cifras de los principales beneficios tributarios para el sector petrolero y los servicios minero-petroleros entre los años 2010 y 2018 y, en la tabla 3, se presentan los ingresos no constitutivos de renta (INCR) y otras deducciones para los mismos sectores mencionados en la tabla 2. En particular, al no contar con los datos desagregados de los INCR y otras deducciones (OD), estos rubros se relacionan como un caso particular de los beneficios tributarios que contribuyen a una reducción del pago de los impuestos. Cabe resaltar que Pardo refiere que:

La DIAN solo considera como beneficios tributarios las deducciones de compra de activos fijos, las rentas exentas y los descuentos tributarios; no obstante, existen otros conceptos pocos claros que permiten a las empresas reducir la base gravable y, por lo tanto, disminuir sus impuestos; estos conceptos son los ingresos no constitutivos de renta (INCR) y otras deducciones (OD). (2018, párr. 11)

En la tabla 2 se puede observar que para el año 2014 los descuentos tributarios entre los sectores analizados alcanzaron los \$131.437 m siendo la cifra más alta durante los periodos analizados; la más baja obedece al año 2016, que reporta los $\$ 24.360 \mathrm{~m}$, debido a la caída de los precios internacionales del petróleo en el año 2015. Por otra parte, se examina que los descuentos tributarios para los sectores analizados son superiores a los de las rentas exentas, siendo más aprovechados por el sector de hidrocarburos a partir del año 2013; según un informe de la DIAN:

Desde 2011 y hasta el año 2016, los beneficios para las empresas estuvieron en promedio alrededor del $3.0 \%$ como proporción del PIB. No obstante, en el año gravable 2017 esta proporción se situó en $1.4 \%$, producto de la eliminación de algunas rentas exentas que fue establecida en la Ley 1819 de 2016 como son: La prestación de servicios de sísmica para el sector de hidrocarburos, La utilidad en la enajenación de predios destinados afines de utilidad pública a que se refieren los literales b) y c) del artículo 58 de la Ley 388 de 1997, entre otros. (2018, p. 67)

En lo que respecta a la compra de activos fijos, esta deducción fue eliminada mediante la Ley 1430 de 2010; de modo que, en la tabla 2 para el sector petrolero no se reportan datos a partir del año 2011, pero en el caso de los servicios minero-petrolero se observan datos, toda vez que aquellas empresas que con anterioridad al 1 de noviembre de 2010 presentaron 
solicitud de contratos de estabilidad jurídica, incluyendo estabilizar la deducción por inversión en activos fijos, continuaron accediendo a este beneficio (Ley 1430, 2010).

Tabla 2. Descuentos tributarios al SECTOR PETROLERo Y SERVicios MineroPETROLEROS EN EL IMPUESTO SOBRE LA RENTA (MILLONES DE PESOS CORRIENTES)

\begin{tabular}{|c|c|c|c|c|c|c|c|c|c|}
\hline \multicolumn{10}{|c|}{ (a) Sector petrolero } \\
\hline Concepto & 2010 & 2011 & 2012 & 2013 & 2014 & 2015 & 2016 & 2017 & 2018 \\
\hline $\begin{array}{l}\text { Descuentos } \\
\text { tributarios }\end{array}$ & 64.018 & 94.341 & 73.112 & 87.199 & 104.072 & 96.712 & 14.845 & 48.064 & 60.440 \\
\hline $\begin{array}{l}\text { Deducción por } \\
\text { inversión en activos } \\
\text { fijos }\end{array}$ & 3.690 .983 & - & - & - & - & - & - & - & - \\
\hline Rentas exentas & 216.451 & 180.374 & 212.042 & 4.772 & 8.308 & 4.556 & 18.222 & 17.484 & 7.045 \\
\hline Subtotal & 3.971 .452 & 274.715 & 285.154 & 91.971 & 112.380 & 101.268 & 33.067 & 65.548 & 67.485 \\
\hline \multicolumn{10}{|c|}{ (b) Sector servicios minero-petroleros } \\
\hline $\begin{array}{l}\text { Descuentos } \\
\text { tributarios }\end{array}$ & 11.195 & 7.199 & 22.677 & 25.603 & 27.365 & 20.607 & 9.515 & 36.445 & 16.266 \\
\hline $\begin{array}{l}\text { Deducción por } \\
\text { inversión en activos } \\
\text { fijos }\end{array}$ & 338.618 & 71.080 & 92.765 & 106.691 & 89.000 & 20.771 & 9.010 & - & - \\
\hline Rentas exentas & 1.799 & 1.313 & 3.110 & 3.311 & 6.804 & 5.031 & 1.075 & 887 & 1.920 \\
\hline Subtotal & 351.612 & 79.592 & 118.552 & 135.605 & 123.169 & 46.409 & 19.600 & 37.332 & 18.186 \\
\hline $\begin{array}{l}\text { Total Sectores } \\
(\mathbf{a}+\mathbf{b})\end{array}$ & 4.323 .064 & 354.307 & 403.706 & 227.576 & 235.549 & 147.677 & 52.667 & 102.880 & 85.671 \\
\hline
\end{tabular}

Fuente: Justicia Tributaria en Colombia (2018) y DIAN (2018).

Frente a la tabla 3, se considera importante contemplar los INCR como mecanismo que permite reducir la renta gravable; al respecto, Justicia Tributaria en Colombia plantea que: "Para los INCR la ley consagra algunos tratamientos fiscales especiales que permiten excluir ciertos ingresos para la determinación de la renta gravable. Entre dichos ingresos se encuentran los dividendos y participaciones, las donaciones, pagos a terceros por alimentación, seguros, el componente inflacionario de los rendimientos financieros y otros" (2018, p. 37).

Se puede observar que en el sector petrolero los INCR para el año 2010 fueron de \$163 mm y para el 2018 fueron de $\$ 3,3$ billones, con un crecimiento de $1.949 \%$; adicionalmente, para el sector servicios minero-petroleros también se refleja un crecimiento del $334 \%$, pasando de $\$ 3 \mathrm{~mm}$ en 2010 a $\$ 13 \mathrm{~mm}$ en 2018. Por otra parte, se puede observar que en el año 2010, las OD para el sector petrolero fueron de $\$ 5$ billones y para 2015 de $\$ 52$ billones teniendo un crecimiento del $805 \%$, y para el sector de servicios minero-petroleros de $207 \%$ en el mismo periodo; especialmente, a raíz de la Reforma Tributaria introducida por la Ley 1819 de 2016, las OD en renta sufrieron varios cambios que, por ende, se ven reflejados en las estadísticas, donde se observa una importante disminución de este rubro 
entre los años 2010 y 2018 en ambos sectores. Para el sector de hidrocarburos la caída fue del $86 \%$ y para el sector de servicios minero-petroleros del $81 \%$.

TABLA 3. INGRESOS NO CONSTITUTIVOS DE RENTA (INCR) Y OTRAS DEDUCCIONES (OD)

AL SECTOR PETROLERO Y SERVICIOS MINERO-PETROLEROS (MILLONES DE PESOS)

\begin{tabular}{|l|c|c|c|r|r|r|r|r|r|}
\hline \multicolumn{7}{|c|}{ Sector petrolero (a) } \\
\hline $\begin{array}{l}\text { Con- } \\
\text { cepto }\end{array}$ & $\mathbf{2 0 1 0}$ & $\mathbf{2 0 1 1}$ & $\mathbf{2 0 1 2}$ & $\mathbf{2 0 1 3}$ & $\mathbf{2 0 1 4}$ & $\mathbf{2 0 1 5}$ & $\mathbf{2 0 1 6}$ & $\mathbf{2 0 1 7}$ & $\mathbf{2 0 1 8}$ \\
\hline INCR & 163.465 & 2.189 .551 & 262.108 & 121.603 & 1.934 .501 & 4.904 .086 & 4.969 .123 & 1.284 .042 & 3.349 .381 \\
\hline OD & 5.801 .503 & 11.084 .682 & 9.576 .754 & 7.547 .640 & 14.800 .315 & 52.531 .872 & 24.035 .221 & 1.014 .472 & 810.385 \\
\hline Subtotal & 5.964 .968 & 13.274 .233 & 9.838 .862 & 7.669 .243 & 16.734 .816 & 57.435 .958 & 29.004 .344 & 2.298 .514 & 4.159 .766 \\
\hline \multicolumn{8}{|c|}{ Sector servicios minero-petroleros (b)* } \\
\hline INCR & 3.162 & 12.169 & 24.973 & 13.446 & 8.747 & 17.481 & 25.218 & 143.726 & 13.720 \\
\hline OD & 356.325 & 473.993 & 619.541 & 352.988 & 669.335 & 1.092 .489 & 531.551 & 140.953 & 67.156 \\
\hline Subtotal & 359.487 & 486.162 & 644.514 & 366.434 & 678.082 & 1.109 .970 & 556.769 & 284.679 & 80.876 \\
\hline $\begin{array}{l}\text { Total } \\
\text { sectores } \\
\text { (a+b) }\end{array}$ & 6.324 .455 & 13.760 .395 & 10.483 .376 & 8.035 .677 & 17.412 .898 & 58.545 .928 & 29.561 .113 & 2.583 .193 & 4.240 .642 \\
\hline
\end{tabular}

Fuente: Justicia Tributaria en Colombia (2018) y DIAN (2018).

* Incluye las actividades de apoyo para la extracción de petróleo y de gas natural, y actividades de apoyo para otras actividades de explotación de minas y canteras.

Recapitulando, se evidenció que en los periodos en que se mostraban altos precios del petróleo $(2011,2012,2013)$ los beneficios por ingresos no constitutivos en renta INCR y OD eran importantes, pero a medida que el precio del petróleo bajó en los años 2015, 2016 y 2017 , los beneficios llegaron a duplicarse, observando que el gobierno no tiene incentivos para gravar a las empresas petroleras pues a estas compañías se les otorgaban una variedad de alivios tributarios en el momento de liquidar sus impuestos lo que, por ende, se refleja en un crecimiento del costo fiscal, de tal manera que entre 2010 y 2016, en opinión de Pardo (2018), el costo fiscal total durante esos siete años ascendió a 23 mil millones de dólares. En razón de lo antes expuesto, estudios en la materia afirman que:

Es difícil comprender por qué las otras deducciones crecieron de manera acelerada en los años estudiados y sobre todo por qué se aceleran justamente en el periodo en que los precios de los minerales y el crudo descienden en el mercado internacional. La caída de los precios conlleva generalmente una disminución de la inversión y por tanto en las deducciones, pero lo que se registra es justamente lo contrario. (Justicia Tributaria en Colombia, 2018, p. 39)

\section{Resultados obtenidos}

En esta sección se expondrán los principales resultados obtenidos a partir del test de Chow. Para comenzar, se comprueba que existen tres cambios estructurales en el comportamiento 
del precio del petróleo Brent, como se observa en la figura 3, los choques se dieron en el primer trimestre de los años 2012, 2014 y 2017.

En la figura 3 se observa que en el eje vertical se encuentra el $\mathrm{F}$ estadístico, el cual permite determinar los puntos de cambios potenciales en una serie de tiempo; las bandas de color rojo situadas en el eje horizontal reflejan el intervalo de confianza, y las líneas verticales punteadas representan los periodos en que se presentaron cambios estructurales. La existencia de cambios en la tendencia del precio promedio del petróleo en el primer trimestre de los años 2012, 2014 y 2017 se explican principalmente por hitos históricos que se mencionarán a continuación:

El primer cambio estructural, según estudios realizados por expertos en la materia, se dio a causa de:

El aumento de los precios del petróleo es un reflejo del equilibrio entre la oferta y la demanda mundial, la mayor parte de la producción de petróleo de Yemen, Siria y Sudán del Sur estaba cerrada y contribuyó a la reducción de la capacidad de producción de repuesto global; para el caso de Sudán el recorte en la oferta obedece a la disputa generada con Sudán del Sur, debido a que a finales de enero de 2012, como resultado de un estancamiento sobre una tarifa de tránsito y otros problemas sociopolíticos, Sudán del Sur cerró toda su producción de petróleo. (IEA, 2012b)

El segundo cambio estructural se originó en el primer trimestre del año 2014, periodo en que el precio promedio del crudo se mantenía estable debido a la alta demanda de petróleo generada por China. Según la Agencia Internacional de la Energía (2014), en la última década, el sólido crecimiento económico y su sed de recursos energéticos han llevado a este país a convertirse en el principal consumidor mundial de energía.

Finalmente, el último cambio estructural se dio en el primer trimestre del año 2017, periodo en que el precio del petróleo empieza a recuperarse a raíz de la desaceleración en el precio presentada a finales de 2014 por un exceso de oferta de petróleo. Expertos en la materia afirman que la recuperación se dio a raíz de:

La menor oferta de la OPEP llegando a niveles mínimos de 32,1 millones de barriles por día, en adelante (Mbpd) por cuenta de la extensión del acuerdo hasta finales de 2018 (incluyendo a Rusia) y la menor producción de Venezuela en 2016-2017; ii) las tensiones geopolíticas de Medio Oriente y la salida de EE.UU. del pacto nuclear con Irán, lo cual estaría disminuyendo las exportaciones de crudo de Irán de los actuales 2,5 Mbpd hacia 1-1,5 Mbpd. (Clavijo, 2018, párr. 1)

En lo que respecta a la relación entre el precio del crudo y los ingresos tributarios se pudo observar que un aumento del precio del petróleo se ve reflejado en un mayor recaudo correspondiente a los impuestos pagados por las compañías vinculadas al sector de hidrocarburos; en contraste con los resultados obtenidos a partir de los tres modelos con los 


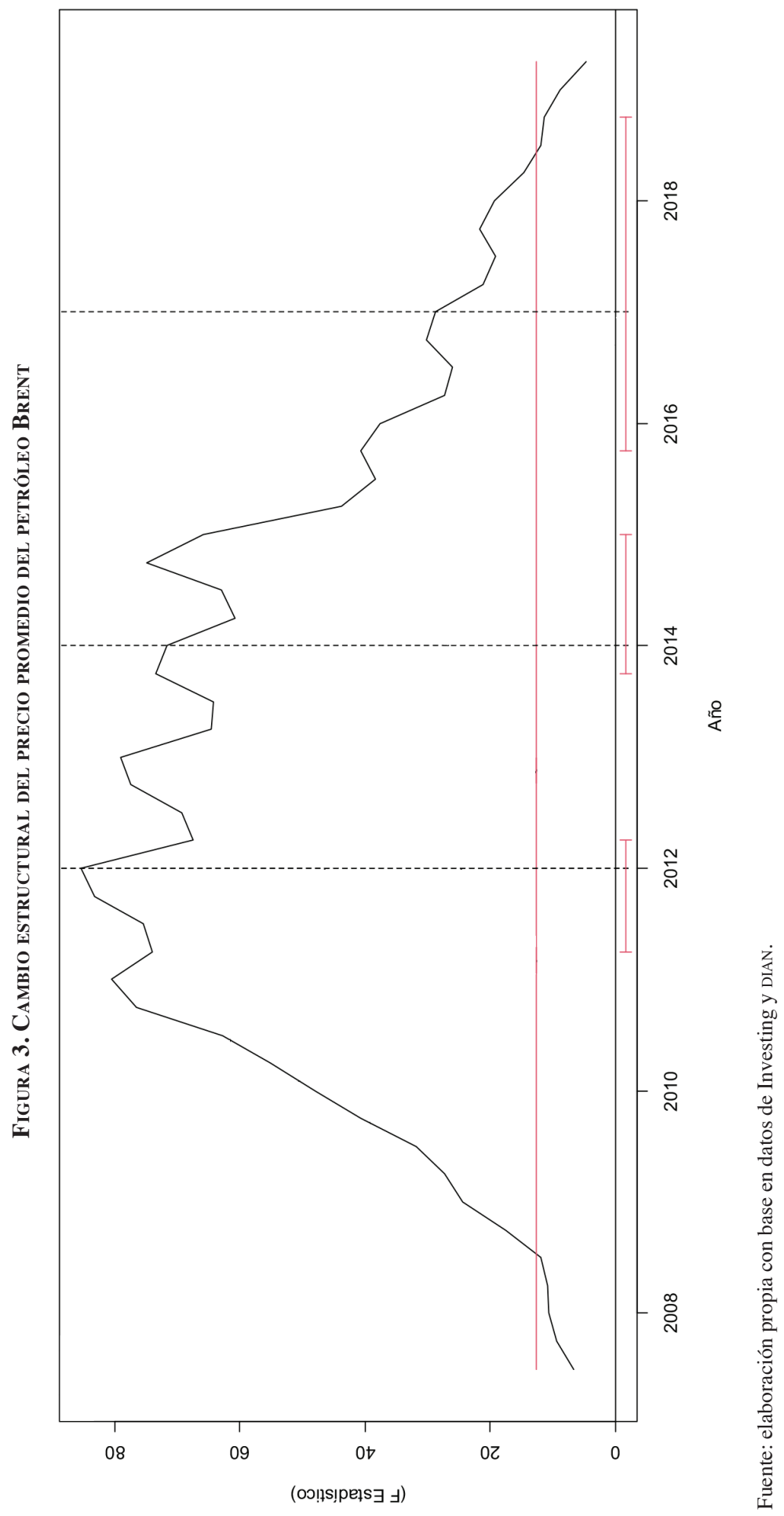

Revista de Derecho Fiscal n. ${ }^{\circ} 19$ • julio-diciembre de 2021 • pp. 109-131 
cuales se realizó el test de Chow, las regresiones reflejaron que existe una relación inversa entre el precio del crudo y los ingresos tributarios, toda vez que a mayor precio del petróleo no hay incentivos para el gobierno de fijar una alta tasa impositiva para las empresas dedicadas a la extracción de hidrocarburos; según Rubiano (2018), "los datos, basados en cifras de la DIAN, lo llevaron a concluir que las empresas no pagan tantos impuestos como deberían, especialmente en los sectores bancario y de minas y petróleos, y que, con cada reforma, lo que se ahorran sube como espuma".

En otras palabras, se evidenció que en los periodos en que se mostraban altos precios del petróleo $(2011,2012,2013)$ los beneficios por ingresos no constitutivos en renta INCR y OD eran importantes, pero a medida que el precio del petróleo bajó en los años 2015, 2016 y 2017, los beneficios llegaron a duplicarse observando que el gobierno no tiene incentivos para gravar a las empresas petroleras pues a estas compañías se le otorgaba una variedad de alivios tributarios en el momento de liquidar sus impuestos que, por ende, se reflejaban en un crecimiento del costo fiscal.

\section{Conclusiones}

La presente investigación tuvo como fin analizar el comportamiento del precio promedio trimestral del petróleo de referencia Brent entre los años 2007-I y 2020-I y su relación con los ingresos tributarios.

Primero, a partir de un análisis en el comportamiento del precio del petróleo Brent se identificó la existencia de tres cambios estructurales en el primer trimestre de los años 2012, 2014 y 2017 que se explican por hechos históricos importantes; para el año 2012, el auge en los precios obedece a un recorte en la oferta de dos países, en el primer caso la mayor parte de la producción de petróleo de Yemen y Siria estuvo cerrada y contribuyó a la reducción de la capacidad de producción de repuesto global; el segundo cambio estructural se originó en el primer trimestre del año 2014 a causa de una estabilidad en el precio promedio del crudo debido a la alta demanda de petróleo generada por China; de acuerdo con la Agencia Internacional de la Energía (2014), el sólido crecimiento económico y su sed de recursos energéticos en la última década han llevado a este país a convertirse en el principal consumidor mundial de energía; finalmente, el tercer cambio estructural se observó en el primer trimestre del año 2017, periodo en que el precio del petróleo empieza a recuperarse a raíz de la desaceleración en el precio presentada a finales de 2014 por un exceso de oferta de petróleo, donde la recuperación se da principalmente por la menor oferta de la OPEP.

Segundo, en lo que respecta a la relación entre el precio del crudo y los ingresos tributarios se pudo observar que a pesar de que un aumento del precio del petróleo se ve reflejado en un mayor recaudo de los ingresos tributarios correspondiente a los impuestos pagados por las compañías vinculadas al sector de hidrocarburos, los resultados de los tres modelos reflejaron que existe una relación opuesta entre el precio del crudo y los ingresos tributarios, toda vez que a mayor precio del petróleo no hay estímulos para el gobierno de 
fijar una alta tasa impositiva para las empresas dedicadas a la extracción de hidrocarburos, ya que se observó una variedad de alivios tributarios a los cuales se acogen las empresas petroleras; sobre todo se observó que, para el año 2010, las otras deducciones para el sector petrolero fueron de $\$ 5$ billones y para 2015 de $\$ 52$ billones, con un crecimiento del $805 \%$, y para el sector de servicios minero-petroleros fue de $207 \%$ para el mismo periodo.

Tercero, se evidenció que en los periodos en que se mostraban altos precios del petróleo $(2011,2012,2013)$ los beneficios por INCR y OD eran importantes, pero a medida que el precio del petróleo bajó en los años 2015, 2016 y 2017 los beneficios llegaron a duplicarse observando que el gobierno no tiene incentivos para gravar a las empresas petroleras, pues, a estas compañías se le otorga una variedad de alivios tributarios en el momento de liquidar sus impuestos que, por ende, se refleja en un crecimiento del costo fiscal.

Finalmente, entre los años 2010 y 2016, el gobierno realizó un esfuerzo por disminuir los beneficios tributarios y las deducciones en renta a las empresas dedicadas al sector de hidrocarburos debido a que se identificó: i) la eliminación de la deducción por inversión en activos fijos establecida en la Ley 1430 de 2010; ii) se excluyeron algunas rentas exentas para las empresas petroleras y se modificó la legislación para las otras deducciones mediante la Ley 1819 de 2016, que tuvo como resultado que entre los años 2010 y 2018 para el sector de hidrocarburos la caída fuera del $86 \%$ y para el sector de servicios minero-petroleros fuera del $81 \%$ en las OD. Sin embargo, este sector continúa recibiendo tratamientos fiscales generosos mediante los INCR, los cuales permiten deducir significativamente la renta gravable. Es de resaltar que al no contar con la información desagregada de los INCR y OD no es posible identificar los rubros que efectivamente corresponden a las deducciones $\mathrm{y}$, por tanto, algunas conclusiones generadas al respecto podrían ser ambiguas.

\section{Referencias}

Achim Zeileis, F. L., Hornik, K. y Kleiber, C. (2002). Strucchange: An R package for testing for structural change in linear regression models. Journal of Statistical Software, 7(2), 1-38. http://www.jstatsoft.org/v07/i02/

Agencia Internacional de Energía (IEA) (2012a). Monthly Oil Marketing, Short-term energy perspective: Market prices and uncertainty report. https://www.eia.gov/ international/analysis/world

Agencia Internacional de Energía (IEA) (2012b). Sudan and South Sudan country analysis summary. https://www.eia.gov/international/analysis/world

Álvarez-Ramírez, J., Álvarez, J. y Rodríguez, E. (2008). Short-term predictability of crude oil markets: A detrended fluctuation analysis approach. Energy Economics, 30, 2645-2656. https://doi.org/10.1016/j.eneco.2008.05.006 
Carcar, M. (2012, 11 de septiembre). El petróleo alcanzó en 2011 el precio más alto en siglo y medio en términos reales. El País. https:/elpais.com/economia/2012/09/11/ actualidad/1347395469_469519.html

Clavijo, S. (2018). Recuperación en precios del petróleo y sus implicaciones. La República. https://www.larepublica.co/analisis/sergio-clavijo-500041/recuperacion-en -precios-del-petroleo-y-sus-implicaciones-2730636

Comisión Económica para América Latina y el Caribe (Cepal) (2017). Tributación en Colombia: reformas, evasión y equidad. Serie Estudios y Perspectivas, 9(35). https:// repositorio.cepal.org/bitstream/handle/11362/43133/1/S1700948_es.pdf

Congreso de Colombia (2010). Ley 1430 de 2010. 29 de diciembre de 2010. Diario Oficial 47.937.

Contraloría General de la República (2016). El impacto de la crisis petrolera en los ingresos del Gobierno Nacional Central. Boletín Macro Fiscal (16), 1-3. https://www. contraloria.gov.co/documents/20194/520989/Bolet\%2525C3\%2525ADn+Macro+Fiscal+16.pdf/0310f5b9-e027-40b3-8502-fa46973fd4b5

Dirección de Impuestos y Aduanas Nacionales (DIAN) (2018). Beneficios tributarios en el impuesto sobre la renta y en el impuesto al valor agregado (IVA) año gravable 2017. https://www.dian.gov.co/dian/cifras/Cuadernos\%20de\%20Trabajo/ Beneficios \%20tributarios \%20en\%20el\%20impuesto\%20sobre $\% 201 \mathrm{a} \% 20 \mathrm{ren}$ ta $\% 20$ y $\% 20$ en $\% 20$ el $\% 20$ impuesto $\% 20$ al $\% 20$ valor $\% 20$ agregado $\% 20$ (IVA) $\% 20$ $\%$ E2\%80\%93\%20a\%C3\%B10\%20gravable\%202017.pdf

Gómez, C. (2015). Más allá de un boom de recursos naturales: efectos de los choques petroleros en la economía colombiana. En Documentos CEDE 012565. Universidad de los Andes, CEDE. https://ideas.repec.org/p/col/000089/012565.html

Hyndman, R., Athanasopoulos, G., Bergmeir, C., Caceres, G., Chhay, L., O’Hara-Wild M.,... Yasmeen, F. (2020)._Forecast: Forecasting functions for time series and linear models_. R package version 8.12. http://pkg.robjhyndman.com/forecast

Justicia Tributaria en Colombia (2018). ¿Y si se eliminan los beneficios al sector extractivo? https://justiciatributaria.co/beneficios-sector-extractivo/

Pardo, A. (2018, 4 de junio). El "regalito" tributario a las compañías de minas y petróleos: US 3.300 millones anuales. Razón Pública. https://razonpublica.com/el-regalito-tributario-a-las-companias-de-minas-y-petroleos-us-3-300-millones-anuales/

Pfaff, B. (2008). Analysis of Integrated and Cointegrated Time Series with R. (2 ed.). Springer. 
Portafolio (2008). Aumento de producción de petróleo para 2012 anuncia OPEP. Portafolio. https://www.portafolio.co/economia/finanzas/aumento-produccion-petro leo-2012-anuncia-opep-335252

Quintana, L. y Mendoza M.(2010). Econometría aplicada utilizando R. Universidad Nacional Autónoma de México, Facultad de Estudios Superiores Acatlán.

R Core Team (2020). R: A language and environment for statistical computing. R Foundation for Statistical Computing. https://www.R-project.org/

Rubiano, M. (2018, 2 de septiembre). Las empresas petroleras y mineras pagan muchos impuestos... ¿o no? El Espectador. https://www.elespectador.com/ noticias/medio-ambiente/las-empresas-petroleras-y-mineras-pagan-muchos-impuestos-o-no-articulo-809824

Savchev, D. y Nason, G. (2018). hwwntest: Tests of White Noise using Wavelets. R package version 1.3.1. https://CRAN.R-project.org/package=hwwntest

Toro, J. y López, D. (2016). El choque petrolero y sus implicaciones en la economía colombiana. Reportes del Emisor, (200), 1-8.

Trapletti,A. y Hornik, K. (2019). Tseries: Time Series Analysis and Computational Finance. $\mathrm{R}$ package version $0.10-47$.

Valencia, O., Zapata, S. y Ramírez, S. (2018). Oil Price Effects on Colombia's Central Government Oil Revenues. Ministerio de Hacienda y Crédito Público.

Velásquez Henao, J. D., Olaya Morales, Y. y Franco Cardona, C. J. (2009). Evidencias de cambios estructurales en el precio promedio mensual del petróleo del West Texas Intermediate (wTI). Cuadernos de Administración, 22(38), 247-266. http:// www.scielo.org.co/scielo.php?script=sci_arttext\&pid=S0120-359220090001 $00012 \& \operatorname{lng}=$ en $\&$ tlng $=$.

Wickham, H. (2016). ggplot2: Elegant Graphics for Data Analysis. Springer-Verlag.

Wooldridge, J. (2015). Introducción a la econometría: un enfoque moderno. Michigan State University.

Fecha de recepción: octubre 31 de 2020

Aprobación par 1: noviembre 9 de 2020

Aprobación par 2: noviembre 18 de 2020 